\title{
Second order active filter design using Operational Trans-conductance Amplifier
}

\author{
Dr.M.Santhanalakshmi ${ }^{1}$ \\ \{ms.ece@ppasgtech.ac.in $\left.{ }^{1}\right\}$ \\ Associate Professor, Department of ECE,PSG Tech Coimbatore ${ }^{1}$
}

\begin{abstract}
An efficient single-stage Folded Cascode operational transconductance amplifier (FC OTA) which features class AB operation is presented. It consumes $32.5 \%$ less power and $45.8 \%$ increase in gain as compared to two stage conventional OTA. Performance parameters of FC OTA is evaluated in 180nm technology using Cadence. As an application of OTA, Second order active filter structure for low pass, high pass and band pass filtering action is designed using the FC OTA. These filters can be used in various applications such as audio processing, image processing etc.
\end{abstract}

Keywords: Active filters, Folded Cascode, Operational Transconductance Amplifier.

\section{Introduction}

Signal Processing has been a major part of the Science and Technology. It is being used in human's day to day life like audio processing and image processing. In the real world, all the physical signals are considerably affected by channel noise. Even in case of communication related circuits, white noise, thermal noise, etc. are the major area of concern to improve the efficiency of modulators and demodulators. Hence, these signals must be filtered using filters like a Low pass filter, High pass filter and band pass filter based on the requirement of the design. There are different techniques to design these Filters. One among them is the use of Folded Cascode Operational Transconductance Amplifiers (OTA) as an active element. This OTA provides high DC gain and larger voltage swing. In conventional Folded Cascode OTAs, the slew rate is limited and so a small modification carried out in conventional OTA results in implemented Folded Cascode OTA, which overcome the limitation on slew rate. It also provides good common mode signal Rejection. So this OTA is utilized for designing the second order active conventional and Butterworth filter.

\section{Existing Work}

In conventional filter design, operational amplifier (op amp) is used as an active element. For design purposes, assume that the op amp is ideal with Av $\square \square$, Rin $\square \square$, Ro $\square 0$, and large amounts of feedback are used to make the filter gain essentially independent of the gain of the operational amplifier. A host of practical filter designs has evolved following this approach. In [1], opamp got replaced by conventional OTA. The problem with conventional OTA was that it operates on limited differential input voltage. In [2], an improved version of a 
fully differential Folded Cascode OTA (operational trans-conductance amplifier), switched at $16 \mathrm{MHz}$ with a single $3 \mathrm{~V}$ supply voltage, is presented. In [3], two different filter structures which use single-ended OTAs and grounded capacitors are discussed. The switching process is performed by current deviation at the OTA output stage, instead of turning off its bias current or interrupting the current path from the power supply. In order to overcome the limitations of conventional OTA, many different types of OTAs are proposed. In [4], a bodydriven (BD) CMOS triode-based fully balanced OTA is discussed that tries to achieve low distortion and linear frequency tuning. As against the gate-driven based OTAs BD-based OTAs operate under a wide range of input voltages. In [5], active RC filter using opamp is discussed. In [6], a single stage Folded Cascode OTA is discussed which provides adaptive biasing. In [7], Recycling Folded OTA is proposed where existing current is reused to perform additional task. All transistors present in the design are supposed to operate in saturation region. It has less figure of merit. In order to overcome this limitation, a micropower single stage OTA in [8] is designed which is used for implementation of second order filters structure.

\section{Conventional Filter Structure}

Filters are the key hardware for a wide variety of circuits. In this work, one single structure is used to construct second order low pass filter, high filter and band pass filter by changing the position of input voltage. The conventional filter structure is shown in Fig.1.

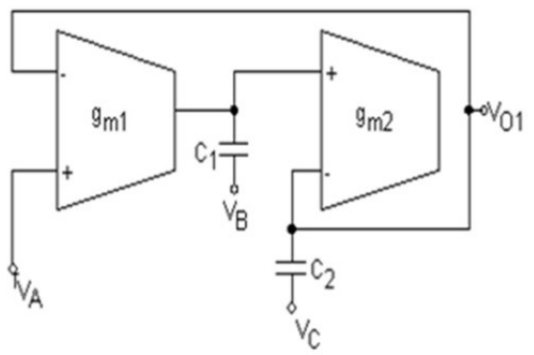

Fig. 1. Conventional Filter Structure

There are 3 input nodes, i.e. $V_{A}, V_{B}$ and $V_{C}$. Different input combinations are used to achieve different filtering actions. At a time, one input is given while others are grounded. The folded Cascode OTA discussed in [8] is used here for filter implementation.

Folded Cascode OTA

The folded cascode (FC) OTA is one of the most used OTAs in both single stage configuration and as the first stage of multistage topologies. This is because of its high gain and reliably large signal swing. Single-stage folded Cascode amplifier able to drive a wide range of capacitive loads is presented. It features class $\mathrm{AB}$ operation and includes powerefficient adaptive biasing techniques, which leads to enhanced dynamic output current and improved Gain Bandwidth Product (GBW). The circuit diagram of Folded Cascode OTA is shown in Fig. 2. 


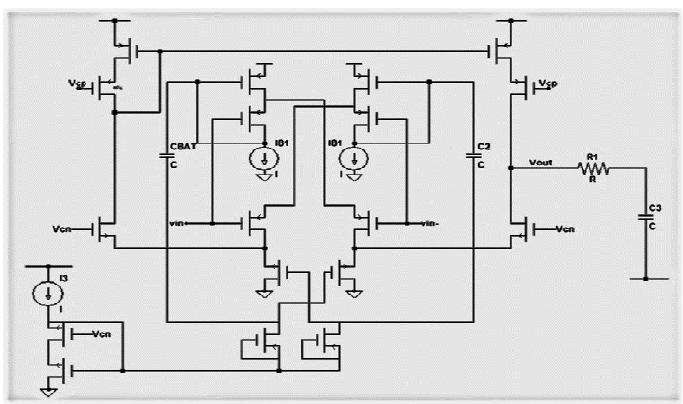

Fig. 2. Folded Cascode OTA.

In this circuit, two pulse sources are given in inverting and non-inverting input. Lead compensation is provided at the output. The values of DC sources are adjusted such that maximum gain can be achieved. The. Current source IB1 and IB2 are used to provide a proper sink to transistors which are fed with inputs. Various performance parameters of the OTA are evaluated to ensure the efficiency of the circuit.

\section{B. Filter Implementation}

To design second order low pass filter, input is given at $V_{A}$ while $V_{B}$ and $V_{C}$ are grounded through capacitor. To design second order high pass filter, input is given at $V_{C}$ while $V_{A}$ and $V_{B}$ are grounded through capacitor and to design second order band pass filter input is given at $V_{B}$ while $V_{A}$ and $V_{C}$ are grounded through capacitor. The alue of capacitors is calculated based on the cutoff frequency.

\section{Butterworth Filter Structure}

In order to extend the application of Folded Cascode OTA, the Butterworth filter structure discussed in [9], as shown in Fig.6 is used to implement low pass filter.

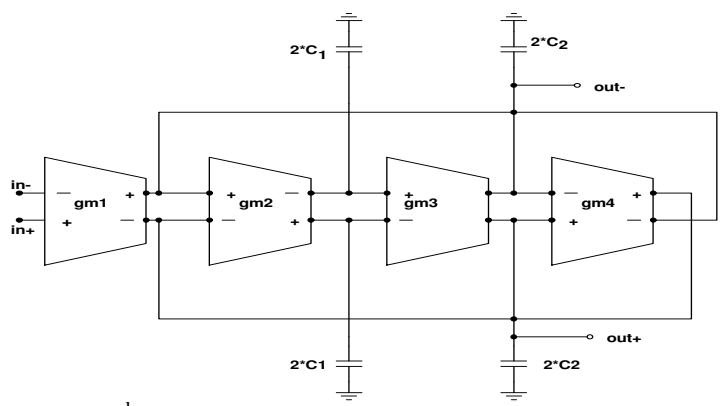

Fig.6. $2^{\text {nd }}$ order Butterworth Low Pass Filter Structure

In this structure four OTA's are used in cascaded fashion to implement second low pass filter. Input is given to first OTA and output is taken from last OTA. 


\section{Result And Analysis}

Folded Cascode OTA is designed in CMOS 180nm technology. Performance parameters of OTAs are summarized in Table 1

Table 1: Performance parameters of OTA

\begin{tabular}{|l|l|l|l|}
\hline S.No & $\begin{array}{l}\text { Performance } \\
\text { Parameters }\end{array}$ & $\begin{array}{l}\text { Two } \\
\text { OTA }\end{array}$ & $\begin{array}{l}\text { Stage } \\
\text { Folded } \\
\text { Cascode } \\
\text { OTA }\end{array}$ \\
\hline 1 & $\begin{array}{l}\text { Differential } \\
\text { Gain }\end{array}$ & $48 \mathrm{~dB}$ & $69.766 \mathrm{~dB}$ \\
\hline 2 & CMRR & $68 \mathrm{~dB}$ & $79.2362 \mathrm{~dB}$ \\
\hline 3 & PSRR & $71 \mathrm{~dB}$ & $79.2312 \mathrm{~dB}$ \\
\hline 4 & Slew Rate & $0.32 \mathrm{~V} / \mathrm{us}$ & $6.79 \mathrm{~V} / \mathrm{us}$ \\
\hline 5 & Power & $80 \mathrm{uW}$ & $54.26 \mathrm{uW}$ \\
\hline
\end{tabular}

Using conventional filter structure, Low pass filters, high pass filter and band pass filter are implemented and their results are shown in Fig.7(a), Fig.7(b), Fig. 7(c) respectively.

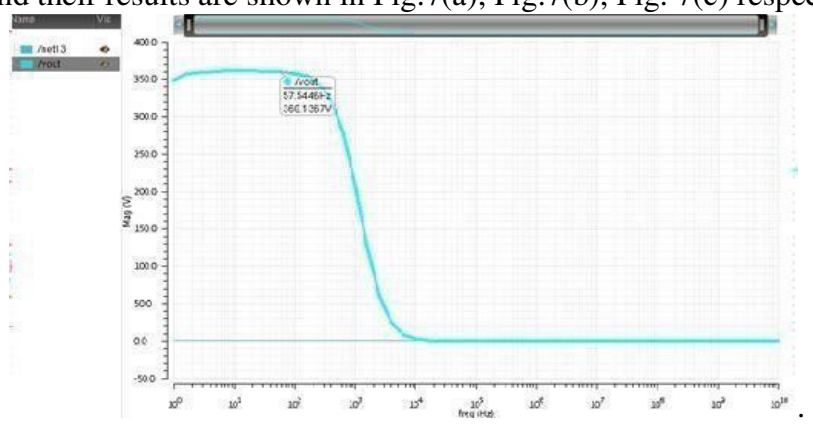

Fig. 7(a). Conventional Low Pass Filter

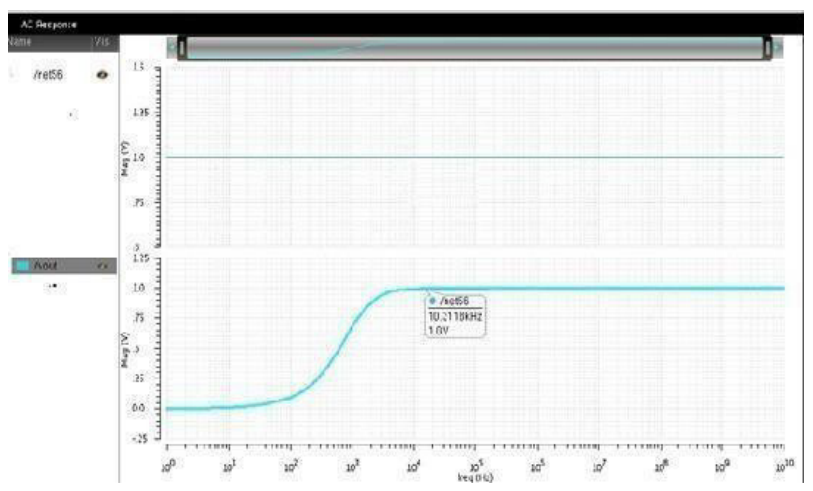

Fig. 7(b). Conventional High Pass Filter 


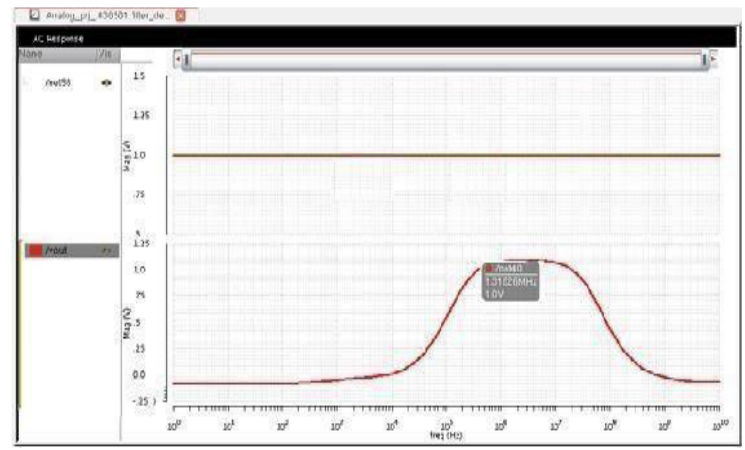

Fig.7(c). Conventional Band Pass Filter

Low pass filter, high pass filter, band pass filter is simulated with cutoff frequency of $1 \mathrm{KHz}, 10 \mathrm{KHz}$ and $100-\mathrm{KHz}-100 \mathrm{MHz}$ respectively. All the three filters are designed for different cut off frequency and values of capacitor is decided based on the chosen cut- off frequency. The simulated result of Second Order Butterworth low pass filter is shown in Fig.8 and it's cut off frequency is found to be $2.5 \mathrm{kHz}$. The power consumption for various filters is calculated and are summarized in Table 2.

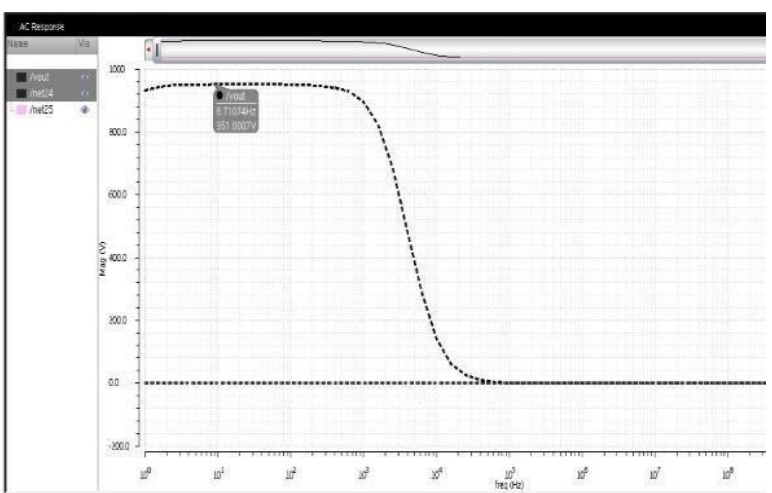

Fig.8. Butterworth Low Pass Filter

Table 2: Parameters of different Filters

\begin{tabular}{|l|l|l|l|l|}
\hline S. No & Filter Type & $\begin{array}{l}\text { Cutoff } \\
\text { Freq. }\end{array}$ & Capacitor & Power \\
\hline 1 & $\begin{array}{l}\text { Low Pass Filter } 1 \mathrm{KHz} \\
\text { Conventional) }\end{array}$ & $1.159 \mu \mathrm{F}$ & $94.56 \mu \mathrm{W}$ \\
\hline 2 & $\begin{array}{l}\text { High Pass Filter } \\
\text { Conventional) }\end{array}$ & $10 \mathrm{KHz}$ & $1.844 \mathrm{nF}$ & $95.71 \mu \mathrm{W}$ \\
\hline 3 & $\begin{array}{l}\text { Band Pass Filter } \\
\text { Conventional) }\end{array}$ & $\begin{array}{l}100 \mathrm{KHz}- \\
100 \mathrm{MHz}\end{array}$ & $1.844 \mathrm{nF}$ & $94.23 \mu \mathrm{W}$ \\
\hline
\end{tabular}




\begin{tabular}{|l|ll|l|l|}
\hline 4. & $\begin{array}{l}\text { Low Pass } \\
\text { (Butterworth) }\end{array}$ & filter $2.5 \mathrm{KHz}$ & $0.463 \mu \mathrm{F}$ & $170.61 \mu \mathrm{W}$ \\
\hline
\end{tabular}

\section{Conclusion}

The Folded Cascode OTA is designed and its performance parameters are calculated. The same OTA is instantiated in filter circuits and the output is recorded for different cutoff frequencies. The value of capacitors used in filter circuit is calculated from the transfer function of respective filter. This work deals with second order Conventional filter structure and Butterworth filter structure that consist of two OTAs and four OTA's respectively.

The folded cascode OTA used here consumes $32.5 \%$ less power than conventional two stage OTA. It also provides improved DC gain of $45.8 \%$ more than two stage OTA. These two parameters emphasize the use of the above said folded Cascode OTA to design second order filters.

In electronics industry, more complex devices such as audio processing devices, video processing devices requires good quality filters. In order to improve the quality of signal and also to remove even minute amount of disturbance, higher order filters are used. In future the concept used in this work can be extended to design third and fourth order filter. Numbers of OTAs used should be as less as possible. Hence this work is efficient in terms of hardware required.

\section{References}

[1] Randall L. Geiger and Edgar Sánchez- Sinencio "Active filter design using Operational Transconductance Amplifiers : A Tutorial “, IEEE Circuits And Devices Magzine, Vol. 1,pp.20-32, March1985.

[2] R. Salinas-Cruz ,G. Espinosa-Flores-V, "A Folded cascode switched OTA based on current deviation", Proceedings of the Fourth IEEE International Caracas Conference on Devices, Circuits and Systems ,2002.

[3] Chun-Ming Chang, Bashir M. Al- Hashimi, Yichuang Sun, and J. Neil Ross, "New High-order filter structure using only single ended OTAS and grounded capacitor" IEEE Transactions On Circuits and Systems-II: Express Briefs, Vol. 51, No. 9, September 2004.

[4] Xuguang Zhang, Ezz I. El-Masry "Novel CMOS OTA Based on Bode driven MOSFET and it's Application to OTA-C Filters" ,IEEE Transactions on Circuits and Systems I: Regular Papers ,Volume: 54 ,Issue: 6, June 2007

[5] Dan-Gabriel I. Stănescu, Petre-Marian T. Nicolae, Alexandru- George I. Dobroiu, Lucian-Dinuț D. Popa,"Designing and testing of a Active RC low pass filter with different quality factors" International Symposium on Fundamentals of Electrical Engineering (ISFEE),2014.

[6] Antonio Lopez-Martin, M. Pilar Garde, Jose M. Algueta, Carlos A. de la Cruz Blas, Ramon G. Carvajal,and Jaime Ramirez-Angulo, "Enhanced Single-Stage Folded Cascode OTA Suitable for Large Capacitive Loads IEEE Transactions on Circuits and Systems II: Express Briefs 2016.

[7] Rida S. Assaad and Jose Silva- Martinez, "The Recycling Folded Cascode: A general enhancement of the Folded Cascode Amplifiers" IEEE Journal of Solid-State Circuits, vol. 44, no. 9, September 2009.

[8] M. Pilar Garde, Antonio Lopez-Martin, Ramón G. Carvajal and Jaime Ramírez-Angulo, "Folded Cascode OTA with $5540 \mathrm{MHz} \cdot \mathrm{pF} / \mathrm{mA}$ FoM 2018, IEEE International Symposium on Circuits and Systems (ISCAS).

[9] Uwe Stehr, Frank Henkel, Lutz Dallüge,and Peter Waldow," A fully differential CMOS integrated 4th order reconfigurable gm-c lowpass filter for mobile communication ", 10th IEEE International Conference on Electronics, Circuits and Systems, ( ICECS )2003. 